\title{
J. MIERCZYŃSKI (Wrocław)
}

\section{SMOOTHNESS OF UNORDERED CURVES \\ IN TWO-DIMENSIONAL STRONGLY COMPETITIVE SYSTEMS}

Abstract. It is known that in two-dimensional systems of ODEs of the form $\dot{x}^{i}=x^{i} f^{i}(x)$ with $\partial f^{i} / \partial x^{j}<0$ (strongly competitive systems), boundaries of the basins of repulsion of equilibria consist of invariant Lipschitz curves, unordered with respect to the coordinatewise (partial) order. We prove that such curves are in fact of class $C^{1}$.

A two-dimensional system of $C^{1}$ ordinary differential equations (ODEs)

$$
\dot{x}^{i}=x^{i} f^{i}(x),
$$

where $f=\left(f^{1}, f^{2}\right): K \rightarrow \mathbb{R}^{2}, K:=\left\{x=\left(x^{1}, x^{2}\right) \in \mathbb{R}^{2}: x^{i} \geq 0\right.$ for $\left.i=1,2\right\}$ is called strongly competitive if

$$
\frac{\partial f^{i}}{\partial x^{j}}(x)<0
$$

for all $x \in K, i, j=1,2, i \neq j$ (see M. W. Hirsch's papers [4] and [5]).

Systems of the form (S) describe communities of two interacting biological species: the function $f^{i}$ represents the per capita growth rate of the $i$ th species. Strong competitiveness means that the growth of each species inhibits the growth of the other.

An important subclass of strongly competitive systems consists of strongly competitive Lotka-Volterra systems of the form

$$
\dot{x}^{i}=x^{i}\left(b_{i}+\sum_{j=1}^{2} a_{i j} x^{j}\right)
$$

1991 Mathematics Subject Classification: Primary 34C30.

Key words and phrases: strongly competitive system of ordinary differential equations, Lotka-Volterra system, d-curve, invariant manifold.

Research supported by KBN grant 2 P03A 03915 (1998-2001). 
where $b_{i} \in \mathbb{R}$ and $a_{i j}<0$ for $i \neq j$. For an introduction to Lotka-Volterra systems and their application in biology see the book [7] by J. Hofbauer and K. Sigmund.

First we introduce some notation and definitions. We write $F=\left(F^{1}, F^{2}\right)$ with $F^{i}(x)=x^{i} f^{i}(x)$. The symbol $D F(x)$ stands for the derivative matrix of the vector field $F$ at $x \in K: D F(x)=\left[\left(\partial F^{i} / \partial x^{j}\right)(x)\right]_{i, j=1}^{2}$. Denote by $\phi=\left\{\phi_{t}\right\}$ the local flow generated on $K$ by $(\mathrm{S}): \phi_{t}\left(x_{0}\right)$ is the value at time $t$ of the unique solution of the initial value problem $\dot{x}^{i}=F^{i}(x)$, $x(0)=x_{0}$. For $x \in K$ denote by $(\sigma(x), \tau(x))$ the largest interval on which the mapping $t \mapsto \phi_{t}(x)$ is defined. We define the backward orbit of $x \in K$ as $O_{\mathrm{b}}(x):=\left\{\phi_{t}(x): t \in(\sigma(x), 0]\right\}$, and its forward orbit by $O_{\mathrm{f}}(x):=\left\{\phi_{t}(x):\right.$ $t \in[0, \tau(x))\}$. The union $O_{\mathrm{b}}(x) \cup O_{\mathrm{f}}(x)=: O(x)$ is the orbit of $x$. We refer to $\left\{\phi_{t}(x): t \in\left(t_{1}, t_{2}\right)\right\}, \sigma(x)<t_{1}<t_{2}<\tau(x)$, as an orbit interval. A set $A \subset K$ is invariant if $O(x) \subset A$ whenever $x \in A$. We write $E$ for the set of equilibria: $x \in E$ if and only if $F(x)=0$.

For $x \in K$ and $t \in(\sigma(x), \tau(x))$ denote by $D \phi_{t}(x)$ the derivative matrix of the mapping $\phi_{t}$ at $x$. It is well known that $t \mapsto D \phi_{t}(x)$ satisfies the matrix linear ODE $\dot{M}=D F\left(\phi_{t}(x)\right) M$ with initial condition $M(0)=$ Id.

Let $K^{\circ}$ be the set of points in $K$ with positive coordinates, that is, the interior of $K$ in $\mathbb{R}^{2}$. Set $\partial K$ to be the boundary of $K$ in $\mathbb{R}^{2}: \partial K=K \backslash K^{\circ}$. From the form of the system (S) it is clear that the sets $\partial K$ and $K^{\circ}$ are invariant. The half-lines $K^{i}:=\left\{x \in K: x^{i}=0\right\}$ are invariant as well.

For a vector $v=\left(v^{1}, v^{2}\right) \in \mathbb{R}^{2}$ we write $v \geq 0$ if $v^{1} \geq 0$ and $v^{2} \geq 0$ (we call such vectors nonnegative). If $v^{1}>0$ and $v^{2}>0$ we write $v \gg 0$ and call such a vector positive. Nonpositive and negative vectors are defined in an analogous way. $v>0$ means $v \geq 0$ and $v \neq 0$. For two points $x, y \in \mathbb{R}^{2}$ we write $x \leq y$ if $y-x \geq 0, x<y$ if $y-x>0$, and $x \ll y$ if $y-x \gg 0$. A set $A \subset K$ is unordered if no two points $x, y \in A$ are ordered by the $<$ relation. For $W \subset \mathbb{R}^{2}$ we denote by $\operatorname{span} W$ the linear span of $W$. For $v \in \mathbb{R}^{2}$ we write $\operatorname{span}_{+} v:=\{\alpha v: \alpha \geq 0\}, \operatorname{span}_{-} v:=\{\alpha v: \alpha \leq 0\}$. $\mathbb{S}^{1}$ denotes the unit circle in $\mathbb{R}^{2}$.

For the system $(\mathrm{S})$ by a $d$-curve we understand an invariant unordered (topological) one-dimensional manifold-with-boundary $D \subset K$. The relevance of this concept lies in the fact that the upper and lower boundaries of the repulsion basin of a repelling set $A \subset K^{\circ}$ are finite families of d-curves and intervals of $K^{i}$; see the paper [6] of M. W. Hirsch. A more detailed analysis of repulsion basins for a generic function $f$ was given in M. S. Holtz' dissertation [8]. E. C. Zeeman and M. L. Zeeman [18] showed that for a class of Lotka-Volterra strongly competitive systems d-curves are convex.

In an arbitrary (possibly infinite) dimension, an analog of d-curves (socalled d-hypersurfaces) is one of the principal tools in investigating strongly monotone dynamical systems (see Hirsch [6] for the finite-dimensional case, 
Takáč [15], [16] for the general case). There is an open problem (posed by Hirsch [6]) of determining conditions under which those hypersurfaces are of class $C^{1}$. What is known in general is that they are Lipschitz. Also, sufficient conditions were given for them to be smooth; see Brunovský [2], Mierczyński [10], [11], Tereščák [17], Benaïm [1]. Recently, a counterexample in dimension three has been found by the author [12].

The purpose of the present paper is to show that d-curves are always manifolds-with-boundary of class $C^{1}$ (Theorem 1 ). The fact that the intersection $D \cap K^{\circ}$ is a one-dimensional $C^{1}$ manifold belongs to the folklore in the theory of strongly monotone dynamical systems (although probably no proof of this fact has appeared in print). Difficulties arise when one considers smoothness at the boundary $D \cap \partial K$. As we shall see, in some cases one cannot make use of classical results but must have recourse to ad hoc arguments.

We begin by stating and proving two preliminary lemmas.

Lemma 1. Let $x \in E \cap K^{\circ}$. Then there exists an eigenvector $w$ of $D F(x)$ with $w^{1} w^{2}<0$, such that for each $u \in \mathbb{R}^{2} \backslash \operatorname{span} w$ there is $T \in \mathbb{R}$ such that $\exp (T \cdot D F(x)) u$ is positive or negative.

P r o of. By the Perron-Frobenius theory (see e.g. Seneta's book [14]) applied to the matrix $\exp (-D F(x))$, the spectrum of $D F(x)$ consists of two simple real eigenvalues, $\varrho_{1}<\varrho_{2}$. Denote normalized eigenvectors corresponding to them by $v, w$. Consequently, $\exp (-t \cdot D F(x)) u /\|\exp (-t \cdot D F(x)) u\|$ tends to $\pm v$ as $t \rightarrow \infty$, unless $u \in \operatorname{span} w$. Further, the Perron-Frobenius theory also states that $v$ can be chosen positive and $w$ has components of opposite signs. One needs now to take $T$ sufficiently close to $-\infty$ or to $\infty$.

LEMma 2. Let $x \in E \cap \partial K \backslash\{0\}$, say $x=\left(x^{1}, 0\right)$ with $x^{1}>0$. Then the matrix $D F(x)$ has the form

$$
\left[\begin{array}{ll}
a & b \\
0 & c
\end{array}\right]
$$

with

$$
a=x^{1} \frac{\partial f^{1}}{\partial x^{1}}(x), \quad b=x^{1} \frac{\partial f^{1}}{\partial x^{2}}(x)<0, \quad c=f^{2}(x) .
$$

Moreover, if $a \geq c$ then for each $u \notin \operatorname{span}(1,0)$ there is $T \in \mathbb{R}$ such that $\exp (T \cdot D F(x)) u$ is positive or negative.

Proof. The first part follows by simple computation. To prove the second part, notice that $(1,0)$ is an eigenvector corresponding to the eigenvalue $a$. Assume first $a>c$. Then $v:=(1,(c-a) / b)$ is an eigenvector corresponding to the eigenvalue $c$. As in the proof of the previous lemma we argue that $\exp (-t \cdot D F(x)) u /\|\exp (-t \cdot D F(x)) u\|$ tends to $\pm v /\|v\|$ as $t \rightarrow \infty$, 
unless $u \in \operatorname{span}(1,0)$. Since $v$ has both components positive, it is enough to take $T$ sufficiently close to $-\infty$ or to $\infty$.

Assume now $a=c$. Then for each $t \in \mathbb{R}$ the matrix $\exp (t \cdot D F(x))$ has the form

$$
e^{a t}\left[\begin{array}{cc}
1 & b t \\
0 & 1
\end{array}\right] .
$$

Therefore it suffices to consider $a=0$. Assume $u^{2}>0$. If $u^{1}>0$ take $T=0$. So, let $u^{1} \leq 0$. One has $(\exp (-t \cdot D F(x)) u)^{1}=u^{1}-b t u^{2} \rightarrow \infty$ as $t \rightarrow \infty$, while $(\exp (-t \cdot D F(x)) u)^{2}=u^{2}$ for all $t \in \mathbb{R}$.

We now formulate and prove our main result.

Theorem 1. Assume that $D$ is a d-curve for a two-dimensional strongly competitive system (S). Then $D$ is a $C^{1}$ manifold (possibly with boundary).

Proof. For $x \in D$ define the tangent cone $\mathcal{C}_{x}:=\{\alpha v \in V: \alpha \geq 0$ and there is a sequence $\left\{x_{n}\right\} \subset D \backslash\{x\}$ such that $x_{n} \rightarrow x$ and $\left(x_{n}-x\right) /\left\|x_{n}-x\right\|$ $\rightarrow v$ as $n \rightarrow \infty\}$. The set $\mathcal{C}_{x}$ is easily seen to be a closed proper superset of $\{0\}$.

At $x \in D \backslash E$ the curve $D$ is locally a portion of the orbit $O(x)$, hence $\mathcal{C}_{x}=\operatorname{span} F(x)$, and the tangent cones $\mathcal{C}_{y}$ depend continuously on $y$ in some relative neighborhood of $x$ in $D$.

Assume that $x \in D \cap E$ and one of the following holds:

(A1) $x \in K^{\circ}$,

(A2) $\quad x \in \partial K$, say $x=\left(x^{1}, 0\right)$ with $x^{1}>0$, and the eigenvalue of $D F(x)$ corresponding to the eigenvector $(1,0)$ either has multiplicity two or is larger than the remaining one.

By Lemma 1 (in case (A1)) or Lemma 2 (in case (A2)) we find a normalized eigenvector $w$ of $D F(x)$ with the property that for each $u \notin \operatorname{span} w$ there is $T \in \mathbb{R}$ such that both components of $\exp (T \cdot D F(x)) u$ are of the same sign. We prove first that $\mathcal{C}_{x} \subset \operatorname{span} w$. Suppose for contradiction that there is $u \in \mathcal{C}_{x} \backslash \operatorname{span} w$. For each $t \in \mathbb{R}$ the mapping $\phi_{t}$ is a local diffeomorphism leaving $x$ fixed, such that its derivative $D \phi_{t}(x)$ equals $\exp (t \cdot D F(x))$. As a consequence, $\exp (t \cdot D F(x)) \mathcal{C}_{x}=\mathcal{C}_{x}$ for all $t \in \mathbb{R}$. In particular, the positive or negative vector $\exp (T \cdot D F(x)) u$ belongs to the tangent cone at $x$. From this we derive that there is a point $y \in D$ in the $\ll$ or $\gg$ relation with $x$, which is in contradiction to the fact that $D$ is unordered.

We have so far proved that $D$ is differentiable at all but (at most) two of its points. Also, $D$ is of class $C^{1}$ on its relatively open subset $D \backslash E$. So, for $x \in D \cap E$ satisfying (A1) or (A2) we already know that the tangent cones $\mathcal{C}_{y}$ are one-dimensional for $y$ in some neighborhood of $x$. We claim that for sequences $\left\{x_{n}\right\} \subset D \backslash\{x\}, x_{n} \rightarrow x$, and $\left\{v_{n}\right\}, v_{n} \in \mathcal{C}_{x_{n}} \cap \mathbb{S}^{1}, v_{n} \rightarrow v$, one has 
$v \in \operatorname{span} \mathcal{C}_{x}$. As $\mathbb{S}^{1}$ is compact, suppose by way of contradiction that there are subsequences (denoted again by $\left\{x_{n}\right\},\left\{v_{n}\right\}$ ) such that $\lim _{n \rightarrow \infty} v_{n}=u \notin$ $\operatorname{span} \mathcal{C}_{x}$. Let $T \in \mathbb{R}$ be such that $\exp (T \cdot D F(x)) u$ is positive or negative. One has $\phi_{T}\left(x_{n}\right) \rightarrow \phi_{T}(x)=x$ and $D \phi_{T}\left(x_{n}\right) v_{n} \rightarrow D \phi_{T}(x) u=\exp (T \cdot D F(x)) u$ as $n \rightarrow \infty$. Consequently, for $N$ sufficiently large, $D \phi_{T}\left(x_{N}\right) v_{N}$ has both components of the same sign. But as $v_{N} \in \mathcal{C}_{x_{N}}$, one has $D \phi_{T}\left(x_{N}\right) v_{N} \in$ $\mathcal{C}_{\phi_{T}\left(x_{N}\right)}$. This yields that close to $\phi_{T}\left(x_{N}\right) \in D$ there is a point $y \in D$ in the $\ll$ relation with the former, a contradiction.

The above technique does not seem to work for the case of $x \in D \cap \partial K$ (say, $x=\left(x^{1}, 0\right)$ ) such that the eigenvalue, $a$, of $D F(x)$ corresponding to the eigenvector $(1,0)$ is smaller than the remaining one, $c$. Assume first that $a<c<0$. Then for each $y$ from some relative neighborhood $U$ of $x$ in $K$ one has $\lim _{t \rightarrow \infty} \phi_{t}(y)=x$. Consequently, we can write $D \cap U=$ $O_{\mathrm{f}}(y) \cup\{x\}$ for some $y \in D \cap U, y \neq x$. By Thms. VIII.2.1 and VIII.3.1 in Hartman [3], $F\left(\phi_{t}(y)\right)$ as well as $\left(x-\phi_{t}(y)\right) /\left\|x-\phi_{t}(y)\right\|$ converge, as $t \rightarrow \infty$, to a (normalized) eigenvector of $D F(x)$, whence $D \cap U$ is $C^{1}$. An analogous reasoning, with $t$ replaced by $-t$, applies when $0<a<c$. Assume now $a<0<c$, and denote by $w$ a normalized eigenvector of $D F(x)$ corresponding to $c$. By the Grobman-Hartman theorem (see e.g. Thm. IX.7.1 in [3]) there is a homeomorphism $h$ of a neighborhood of $x$ into a neighborhood of 0 in $\mathbb{R}^{2}$ sending orbit intervals of the dynamical system $\phi$ to orbit intervals of the linearized system $\dot{\xi}=D F(x) \xi$. For the latter the invariant one-dimensional topological manifolds-with-boundary for which 0 is a boundary point are $\operatorname{span}_{+}(1,0), \operatorname{span}_{-}(1,0), \operatorname{span}_{+} w$ and $\operatorname{span}_{-} w$. The inverse images of those manifolds under $h$ are portions of (local) unique stable or unstable $C^{1}$ manifolds, $M^{\mathrm{s}}(x), M^{\mathrm{u}}(x)$, at $x$ (for the theory of stable and unstable manifolds see e.g. Kelley [9]). As a consequence, $D$ is of class $C^{1}$ in a neighborhood of $x$.

The remaining cases are those of one of $a$ or $c$ equal to zero. We have either $a=0$ and $c>0$, or $a<0$ and $c=0$. Assume the former. Then there exist a locally invariant $C^{1}$ one-dimensional center manifold $M^{\mathrm{c}}(x)$ tangent at $x$ to $(1,0)$ and a locally invariant $C^{1}$ one-dimensional unstable manifold $M^{\mathrm{u}}(x)$ tangent at $x$ to $w$. (A set $A$ contained in a neighborhood $U$ of $x$ is locally invariant if $O(x) \cap U \subset A$ for each $x \in A$.) The manifold $M^{\mathrm{u}}(x)$ is unique, whereas $M^{\mathrm{c}}(x)$ is not, in general. The dynamical system $\phi$ restricted to a neighborhood $U$ of $x$ has local product structure (cf. Palis and Takens [13]): there is a homeomorphism $h$ of $U$ into a neighborhood of 0 in $\mathbb{R}^{2}$ carrying orbit intervals of $\phi$ to orbit intervals of the dynamical system generated by the two uncoupled ODEs $\dot{\xi}^{1}=g\left(\xi^{1}\right), \dot{\xi}^{2}=c \xi^{2}$, where $g(0)=$ $g^{\prime}(0)=0$. Also, $h$ carries some $M^{\mathrm{c}}(x)$ into $\left\{\left(\xi^{1}, 0\right)\right\}$ and carries $M^{\mathrm{u}}(x)$ into $\left\{\left(0, \xi^{2}\right)\right\}$. Further, although $h$ is only continuous, different center manifolds at $x$ correspond via $h$ in a one-to-one way to different locally invariant sets 
tangent at 0 to $\left\{\left(\xi^{1}, 0\right)\right\}$. So, in the $\xi$-coordinates any topological locally invariant one-dimensional manifold-with-boundary having 0 as a boundary point either is a portion of $\left\{\left(0, \xi^{2}\right)\right\}$ or is tangent at 0 to $\left\{\left(\xi^{1}, 0\right)\right\}$. From this it follows that locally $D$ is either $M^{\mathrm{u}}(x)$ or some center manifold at $x$. Both are $C^{1}$.

The case $a<0$ and $c=0$ is treated in an analogous way: There is a unique one-dimensional stable manifold $M^{\mathrm{s}}(x)$ tangent at $x$ to $(1,0)$, and a one-dimensional center manifold $M^{\mathrm{c}}(x)$. Since the half-line $K^{1}$ is invariant and tangent at $x$ to $(1,0)$, there is a neighborhood $U$ of $x$ such that $K^{1} \cap U=$ $M^{\mathrm{c}}(x) \cap U$. Using the local product structure argument as in the previous paragraph we conclude that $D \cap U$ is a $C^{1}$ center manifold.

\section{References}

[1] M. Benaïm, On invariant hypersurfaces of strongly monotone maps, J. Differential Equations 137 (1997), 302-319.

[2] P. Brunovský, Controlling nonuniqueness of local invariant manifolds, J. Reine Angew. Math. 446 (1994), 115-135.

[3] P. Hartman, Ordinary Differential Equations, Wiley, New York, 1964.

[4] M. W. Hirsch, Systems of differential equations which are competitive or cooperative. I. Limit sets, SIAM J. Math. Anal. 13 (1982), 167-179.

[5] - Systems of differential equations that are competitive or cooperative. II. Convergence almost everywhere, ibid. 16 (1985), 423-439.

[6] - Systems of differential equations which are competitive or cooperative. III. Competing species, Nonlinearity 1 (1988), 51-71.

[7] J. Hofbauer and K. Sigmund, The Theory of Evolution and Dynamical Systems, London Math. Soc. Stud. Texts 7, Cambridge Univ. Press, Cambridge, 1988.

[8] M. S. Holtz, The topological classification of two dimensional cooperative and competitive systems, Ph.D. dissertation, Univ. of California, Berkeley, 1987.

[9] A. Kelley, The stable, center-stable, center, center-unstable, unstable manifolds, J. Differential Equations 3 (1967), 546-570; reprinted as Appendix C in the book: R. Abraham and J. W. Robbin, Transversal Mappings and Flows, Benjamin, New York, 1967, 134-154.

[10] J. Mierczyński, The $C^{1}$ property of carrying simplices for a class of competitive systems of ODEs, J. Differential Equations 111 (1994), 385-409.

[11] -, On smoothness of carrying simplices, Proc. Amer. Math. Soc. 127 (1999), $543-551$.

[12] _- Smoothness of carrying simplices for three-dimensional competitive systems: $A$ counterexample, Dynam. Contin. Discrete Impuls. Systems, in press.

[13] J. Palis and F. Takens, Topological equivalence of normally hyperbolic dynamical systems, Topology 16 (1977), 335-345.

[14] E. Seneta, Non-negative Matrices and Markov Chains, 2nd ed., Springer Ser. Statist., Springer, New York, 1981.

[15] P. Takáč, Convergence to equilibrium on invariant d-hypersurfaces for strongly increasing discrete-time semigroups, J. Math. Anal. Appl. 148 (1990), 223-244.

[16] - Domains of attraction of generic $\omega$-limit sets for strongly monotone discretetime semigroups, J. Reine Angew. Math. 423 (1992), 101-173. 
[17] I. Tereščák, Dynamics of $C^{1}$ smooth strongly monotone discrete-time dynamical systems, preprint.

[18] E. C. Zeeman and M. L. Zeeman, On the convexity of carrying simplices in competitive Lotka-Volterra systems, in: Differential Equations, Dynamical Systems, and Control Science, Lecture Notes in Pure and Appl. Math. 152, Dekker, New York, 1994, 353-364.

Janusz Mierczyński

Institute of Mathematics

Wrocław University of Technology

Wybrzeże Wyspiańskiego 27

50-370 Wrocław, Poland

E-mail: mierczyn@banach.im.pwr.wroc.pl 